\title{
Fatty acid sensitivity, intake of high-fat foods, gene polymorphism, and body mass
}

\section{Abstract}

Taste perception is the main biological determinant of food choice. It has thus been hypothesized that fatty acid sensitivity may affect fat intake. The aim of this study was to examine the relationship between fatty acid sensitivity, frequency of consumption of high-fat products, polymorphism of genes encoding proteins involved in fat taste perception, and body mass.

421 people aged 20-40 were enrolled in Poznań, Poland. Body composition was measured using a Bod Pod. The frequency of consumption of high-fat foods was analyzed using an application for mobile devices based on the ecological momentary assessment approach. Food intake was assessed with dietary records. Salad dressings with varying concentrations of canola oil (from $2.5 \%$ to $40.0 \%$ ) were used as stimuli to test fatty acid sensitivity. The individuals were then divided into groups with higher and lower fatty acid sensitivity. Lower sensitivity means that individuals were able to distinguish samples when the oil concentration exceeded 20\%. Genotyping of rs1761667 (CD36), rs1573611 (FFARI), and rs17108973 (FFAR4) was performed using TaqMan probes.

$57 \%$ men and $61 \%$ women had higher sensitivity to fatty acids. Higher fatty acid sensitivity was associated with the GG genotype of CD36 $(\mathrm{OR}=2.05, \mathrm{p}<0.05)$. People with different taste sensitivity did not differ in their frequency of consumption of high-fat foods or in their macronutrient intake. There was no association between body mass index (BMI) and fatty acid sensitivity, but people with BMI values below 25 more often ate high-fat products with favorable lipid profiles and less often ate meat high-fat products than subjects with BMI values over 25 ( $\mathrm{p}<0.001$ and $\mathrm{p}<0.05$, respectively). There was no association between $C D 36$ or FFAR4 genotype and fat intake or frequency of consumption of high-fat foods. People with the minor FFARI allele ate sweet high-fat products less often than major allele homozygotes $(\mathrm{p}<0.05)$. Moreover, women ate high-fat products with favorable lipid profiles and sweet and savory high-fat products more frequently than men $(\mathrm{p}<0.05, \mathrm{p}<0.001$, and $\mathrm{p}<0.01)$, but men ate meat high-fat products more frequently than women $(\mathrm{p}<0.01)$.

Concluding, fatty acid sensitivity is associated with polymorphism of the CD36 gene. The frequency of consumption of high-fat foods depends on sex, but not on fatty acid sensitivity, BMI, or CD36 variants.

The project was financed by a National Science Centre award (decision number grant no. 2014/15/B/NZ9/02134).

\section{Conflict of Interest}

There is no conflict of interest 